\title{
Hybrid Particle-Continuum Simulations of Nonequilibrium Hypersonic Blunt-Body Flowfields
}

\author{
Thomas E. Schwartzentruber, $\stackrel{*}{-}$ Leonardo C. Scalabrin,, \pm and Iain D. Boyd $\stackrel{\ddagger}{ \pm}$ \\ University of Michigan, Ann Arbor, Michigan 48109
}

DOI: $\underline{10.2514 / 1.30216}$

\begin{abstract}
Hypersonic blunt-body flowfields containing a mixture of continuum and nonequilibrium flow are investigated using a modular particle-continuum numerical method. The modular particle-continuum method solves the Navier-Stokes equations in near-equilibrium regions and uses the direct simulation Monte Carlo method in nonequilibrium regions. Hypersonic flow of nitrogen over a two-dimensional cylinder at a global Knudsen number of 0.01 is simulated for a range of Mach numbers using the modular particle-continuum method as well as full direct simulation Monte Carlo and full Navier-Stokes algorithms. For these conditions, Navier-Stokes simulations significantly overpredict the local shear stress and also overpredict the peak heating rate by $5-10 \%$ when compared with direct simulation Monte Carlo results. The direct simulation Monte Carlo method also predicts faster wake closure and $10-15 \%$ higher temperatures in the immediate wake region. The modular particle-continuum code is able to accurately reproduce the flowfield results, local velocity distributions, and surface properties obtained using the direct simulation Monte Carlo method up to 2.8 times faster. It is found that when using the modular particlecontinuum method, particle simulation of the bow-shock interior is not necessary for accurate prediction of surface properties. However, particle simulation is required for the boundary-layer and near-wake regions
\end{abstract}

\section{Introduction}

A CCURATE numerical simulation of the entry of a hypersonic vehicle into a planetary atmosphere is vital to the successful design of such vehicles. Reproducing such high-energy, low-density flows in wind-tunnel experiments is very expensive and for many flows is currently not even possible. Such spacecraft generally transition from high-speed rarefied conditions to lower-speed continuum conditions. Furthermore, at intermediate altitudes within a mostly continuum flow, there may be local regions of rarefied (or nonequilibrium) flow generated by both the rapid expansion in the wake of the spacecraft and by strong gradients in shock waves and boundary layers. Ultimately, it is the flow conditions inside the wake and boundary layer that determine the drag and heat transferred to the spacecraft surface. Thus, it is important that these regions be simulated accurately using an appropriate physical model.

\section{A. Motivation}

When continuum conditions are present, the Navier-Stokes (NS) equations may be solved using algorithms from computational fluid dynamics (CFD). Although very efficient, solution of the NS equations in nonequilibrium regions can lead to significant error in flowfield and surface properties. The most popular numerical method for simulating high-speed nonequilibrium flow is the direct simulation Monte Carlo (DSMC) method developed by Bird [1]. The DSMC method is accurate in all flow regimes but requires the computational cell size and time step to be on the order of the local mean free path and mean free time, respectively. Thus, DSMC

Presented as Paper 3602 at the 9th AIAA Joint Thermophysics and Heat Transfer Conference, San Francisco, CA, 5-8 June 2006; received 2 February 2007; revision received 14 May 2007; accepted for publication 16 May 2007. Copyright $\odot 2007$ by Thomas E. Schwartzentruber, Leonardo C. Scalabrin, and Iain D. Boyd. Published by the American Institute of Aeronautics and Astronautics, Inc., with permission. Copies of this paper may be made for personal or internal use, on condition that the copier pay the $\$ 10.00$ per-copy fee to the Copyright Clearance Center, Inc., 222 Rosewood Drive, Danvers, MA 01923; include the code 0887-8722/08 \$10.00 in correspondence with the CCC.

${ }^{*}$ Graduate Student, Department of Aerospace Engineering, 1320 Beal Avenue; schwartt@umich.edu. Student Member AIAA.

${ }^{\dagger}$ Graduate Student, Department of Aerospace Engineering, 1320 Beal Avenue; 1scalabr@umich.edu. Student Member AIAA.

†Professor, Department of Aerospace Engineering, 1320 Beal Avenue; iainboyd@umich.edu. Associate Fellow AIAA. becomes very computationally expensive as the flow nears continuum conditions. However, it is precisely under these continuum conditions that the NS equations can be solved efficiently without the same restriction on cell size or time step. A review of numerical simulations using both methods and their agreement with various hypersonic blunt-body experiments is presented in [2]. One conclusion of this review paper is that although the NS results generally match DSMC and experimental data in the forebody flow, they deviate significantly from DSMC and the experiment in the near wake for both surface and flow features. To obtain more accurate results in the near-wake region without resorting to a full DSMC simulation, "zonally decoupled" DSMC-NS simulations [3] have been performed. Here, the forebody flow is solved separately using a NS solver and the exit-plane solution is specified as the inflow condition to a decoupled DSMC simulation of the entire wake region. Results of the zonally decoupled simulations agree well with full DSMC solutions in the wake region, while requiring only half of the computational resources (CPU time and memory) [3]. Comparison between zonally decoupled, full DSMC, and full NS simulations also emphasizes the importance of modeling rarefied effects such as thermal nonequilibrium and slip flow to predict surface properties and wake closure. Using a similar decoupled approach, the flow about the Mars sample return orbiter was simulated at both flight conditions [4] and for a wind-tunnel experiment [5]. The interface between the NS and DSMC regions was taken as a plane cutting through the aeroshell lip. Although this practical approach was much more efficient than a full DSMC simulation, in one simulation, 20 million cells were used in the wake region. This required 200 million particles taking $18 \mathrm{~GB}$ of core memory, and it is reported that regions of the flow may still be underresolved [5]. Thus, there is substantial efficiency to be gained if the DSMC method is restricted only to that portion of the flowfield in which significant nonequilibrium effects are felt.

\section{B. Coupled Hybrid Methods}

Various researchers have proposed hybrid numerical methods that adaptively reposition the DSMC-NS interface during the simulation and couple the two solvers by transferring information across this interface. Reference [6] presents a discussion of the major considerations involved, as well as a summary of published work on such coupled schemes. One problematic aspect is the transfer of information from particle to continuum cells and the inherent statistical scatter in that information. Some researchers determine the 
average flux of mass, momentum, and energy carried by DSMC particles as they cross the interface and impose this as the macroscopic flux into the neighboring NS cell. Such flux-based coupling was used by Hash and Hassan $[\underline{7}, \underline{8}]$ to couple the DSMC method with a NS solver for simulations of Couette flow and hypersonic flow over a blunted cone. More recently, Wijesinghe et al. [9] also used flux-based coupling to embed a DSMC solver in the finest level of an adaptive mesh and algorithmic refinement scheme for the Euler equations. Although flux-based coupling may seem a natural way to couple particle and continuum solvers, the statistical scatter inherent in determining average flux quantities in a DSMC simulation is very high. In regions in which the interface is aligned parallel with the flow direction, the vast majority of particles move along the interface, compared with the relatively few that cross the interface. As a result of this small sample size for DSMC flux quantities crossing such an interface, the statistical scatter becomes far too large to impose on the NS domain [10]. An alternate method of information transfer between DSMC and NS domains is to use state-based coupling. Here, particles inside a DSMC cell near the interface are averaged to obtain a macroscopic state such as bulk velocity, temperature, and density. The statistical scatter involved in obtaining state quantities is much smaller than that associated with obtaining flux quantities [6]. The averaged macroscopic state is then used as a standard boundary condition for the NS domain. Roveda et al. [11] used state-based coupling to combine the DSMC method with an Euler solver to simulate moving 1-D shock waves and 2-D unsteady slit flow [12]. Although use of state-based coupling involved less statistical scatter, their method was time-accurate and particle information had to be averaged at each time step. This significantly constrained the number of samples used for the average. To deal with this, the authors employed a novel algorithm to effectively "clone" particles near the interface to reduce the statistical scatter transferred to the continuum domain. Wang and Boyd [14] used the information preservation (IP) scheme [13] that, in addition to preserving microscopic information, also preserves macroscopic information for each DSMC simulation particle to reduce the statistical scatter. Although successful for certain 2-D flows, when applied to 1-D normal shock waves, it was found that the IP scheme produced an incorrect postshock state and a shock wave that was too thin. A new formulation for the IP energy flux [15] was able to remedy these problems somewhat, but at large computational expense.

The hybrid particle-continuum method used in this paper is designed specifically for steady-state hypersonic flows. The algorithm was first validated for 1-D normal shock waves [16] and has recently been extended and modified for 2-D flows [17]. By restricting application to steady-state flows, the modular particlecontinuum (MPC) method is able to use temporal averaging combined with state-based coupling to effectively manage statistical scatter. Focusing on steady-state flows also allows use of an implicit NS solver, enabling timescale decoupling that results in significant computational savings. Finally, state-based coupling allows for information transfer to be handled by the standard boundary procedures used by both DSMC and NS solvers. Taking advantage of these ideas, the hybrid DSMC-NS method used in this paper is implemented in a novel modular way. The algorithm combines existing state-of-the-art DSMC and NS codes (virtually unmodified) into one MPC numerical method. Details of the MPC method are briefly outlined and the method is then used to analyze nonequilibrium effects in simple hypersonic blunt-body flows.

\section{Flow Conditions Investigated}

Flow conditions are chosen to lie in the transitional regime containing regions of both continuum flow and regions that exhibit significant nonequilibrium effects. Such effects include a strong bow shock, thermal nonequilibrium, velocity slip, and temperature jump on surfaces and rarefied recirculating flow in the wake. Specifically, two-dimensional hypersonic flow of $\mathrm{N}_{2}$ gas over an 8-cm-diam cylinder at an altitude of approximately $70 \mathrm{~km}$ is investigated. Using the U.S. Standard Atmosphere, this altitude corresponds to a freestream density $\rho=7.48 \times 10^{-5} \mathrm{~kg} / \mathrm{m}^{3}$ and temperature $T=217.45 \mathrm{~K}$. Assuming the gas is composed only of $\mathrm{N}_{2}$, these conditions correspond to a number density $n=1.61 \times 10^{21} 1 / \mathrm{m}^{3}$ and pressure $p=4.83 \mathrm{~Pa}$, resulting in a global Knudsen number of 0.01 . To investigate varying degrees of nonequilibrium flow, the freestream velocity is set to 902,1804 , and $3608 \mathrm{~m} / \mathrm{s}$, resulting in Mach 3, 6, and 12 flows, denoted as cases M3, M6, and M12. For each simulation, the cylinder wall temperature is assumed constant at 300, 500, and $1000 \mathrm{~K}$ for cases M3, M6, and M12, respectively.

\section{Modular Particle-Continuum Numerical Method}

The MPC method involves the use of existing DSMC and NS algorithms, a procedure to determine when each method should be used, and a procedure to transfer information between DSMC and NS regions. This section briefly describes these procedures. Complete details of the MPC algorithm can be found in [17].

\section{A. NS and DSMC Algorithms}

Existing state-of-the-art DSMC and NS codes are incorporated into the MPC method. The DSMC portion is simulated using MONACO [18], a general cell-based implementation of the DSMC method. The variable-hard-sphere (VHS) collision model is employed, which results in the following macroscopic viscosity model [1] :

$$
\mu=\mu_{\text {ref }}\left(\frac{T}{T_{\text {ref }}}\right)^{\omega}, \quad \mu_{\text {ref }}=\frac{15 \sqrt{\pi m k T_{\text {ref }}}}{2 \pi d_{\text {ref }}^{2}(5-2 \omega)(7-2 \omega)}
$$

All numerical results presented in this paper are for diatomic nitrogen $\mathrm{N}_{2}$ with a reference diameter of $d_{\text {ref }}=4.17 \times 10^{-10} \mathrm{~m}$ at $T_{\text {ref }}=273 \mathrm{~K}$. The power-law exponent $\omega$ is set equal to $0.75, m$ is the mass of an $\mathrm{N}_{2}$ molecule, and $k$ is the Boltzmann constant. MONACO employs the variable rotational-energy-exchange probability model of Boyd [19], in which the reference temperature for rotational energy exchange is specified as $91.5 \mathrm{~K}$ and the maximum rotational collision number is 18.1. Energy transfer to vibrational modes is not considered. The DSMC method assumes diffuse reflection and full thermal accommodation at the cylinder wall. The same mesh is used for all DSMC, NS, and MPC simulations of all cases. The mesh is structured and consists of 600 evenly spaced cells along the surface of the cylinder and 300 cells in the radial direction. In the forebody, the cell size is clustered toward the cylinder surface. For all cases, the cell size is verified to be less than the local mean free path throughout the domain. This constraint is a requirement of the DSMC method and is more than sufficient for an accurate NS simulation. The reference particle weight is set to obtain a minimum of 20 particles per cell. Constant DSMC time steps of $5 \times 10^{-8} \mathrm{~s}$ are used for both the $\mathbf{M 3}$ and $\mathbf{M 6}$ cases, whereas a time step of $2 \times 10^{-8}$ s is used for case M12. These time steps are less than one-half of the shortest mean free time anywhere in each simulation.

The continuum portion is simulated using the LeMANS code [20]. In continuum NS regions, LeMANS assumes that rotational and translational energy modes can be described by a single temperature $T$. The vibrational energy mode is not considered. The resulting governing equations are the two-dimensional, laminar, compressible Navier-Stokes equations. The viscosity is modeled using Eq. (1) to exactly match the viscosity model used in DSMC. This is important not only at DSMC-NS interfaces (for information transfer) but also whenever comparing DSMC and NS flowfield results and surface properties. LeMANS solves this set of equations using a finite volume formulation. The inviscid fluxes between the mesh volumes are discretized using a modified form of the Steger-Warming fluxvector splitting, which is less dissipative and adequate to calculate boundary layers. The scheme switches back to the original form of Steger-Warming near shock waves. The viscous terms are calculated using the values of properties at the cell centers and at the nodes. Wall boundaries employ a no-slip condition and inflow and outflow boundaries are treated as supersonic. Finally, the time integration is performed using a point-implicit method. Specific details of the LeMANS code are contained in [20]. 


\section{B. Continuum Breakdown and Interface Location}

Ultimately, the accuracy of a hybrid particle-continuum method relies on the proper positioning of the DSMC-NS interfaces. The interface must lie in near-equilibrium regions in which solution of the NS equations will introduce minimal or no error. Typically, particle and continuum regions are determined by applying a continuum breakdown parameter to the flowfield. The MPC method used in this paper employs the gradient-length Knudsen number:

$$
K n_{\mathrm{GL}-Q}=\frac{\lambda}{Q_{\text {local }}}|\nabla Q|
$$

where $Q$ represents local flow quantities such as density $\rho$, temperature $T$, or velocity magnitude $|V|$, and $\lambda$ is the local mean free path. In low-speed regions of the flow, it is useful to normalize the velocity gradient by the local speed of sound $a$, which can be implemented by setting $Q_{\text {local }}=\max (|V|, a)$. It has been shown for flows representative of hypersonic reentry problems [22] and for 1-D normal shock waves [16] that in regions of the flowfield in which $K n_{\mathrm{GL}}<0.05$, the discrepancy between a NS and DSMC solution is less than $5 \%$. Thus, these regions could be solved using a NS solver with little error. As suggested in [16] and detailed in [17], a condition of thermal equilibrium is added to the definition of continuum breakdown, because Eq. (2) does not account for this. The final value used to quantify the degree of local continuum breakdown, including thermal nonequilibrium, is then taken as

$$
K n_{\mathrm{GL}}=\max \left(K n_{\mathrm{GL}-\rho}, K n_{\mathrm{GL}-T}, K n_{\mathrm{GL}-|V|}, 5 \times \frac{T_{\mathrm{TRA}}-T_{\mathrm{ROT}}}{T_{\mathrm{ROT}}}\right)
$$

Thus, if the translational temperature is more than $1 \%$ larger than the rotational temperature, then $K n_{\mathrm{GL}}$ is given a value greater than 0.05 and the region is considered to be a nonequilibrium one. The MPC method begins with a NS solution, and Fig. 1a (top) plots the value of $K n_{\mathrm{GL}}$ from Eq. (3) applied to this initial NS solution for the M6 case. Figure 1a (bottom) also plots the error in this NS solution compared with a full DSMC solution, which is given by

$$
E=\max \left|\frac{Q_{\mathrm{NS}}-Q_{\mathrm{DSMC}}}{Q_{\mathrm{DSMC}}}\right|
$$

where $Q=\rho, T$ or $|V|$.

Because the contours depict a maximum of several quantities, any apparent discontinuities in Fig. 1a are regions in which a new quantity has become dominant. As expected, the breakdown

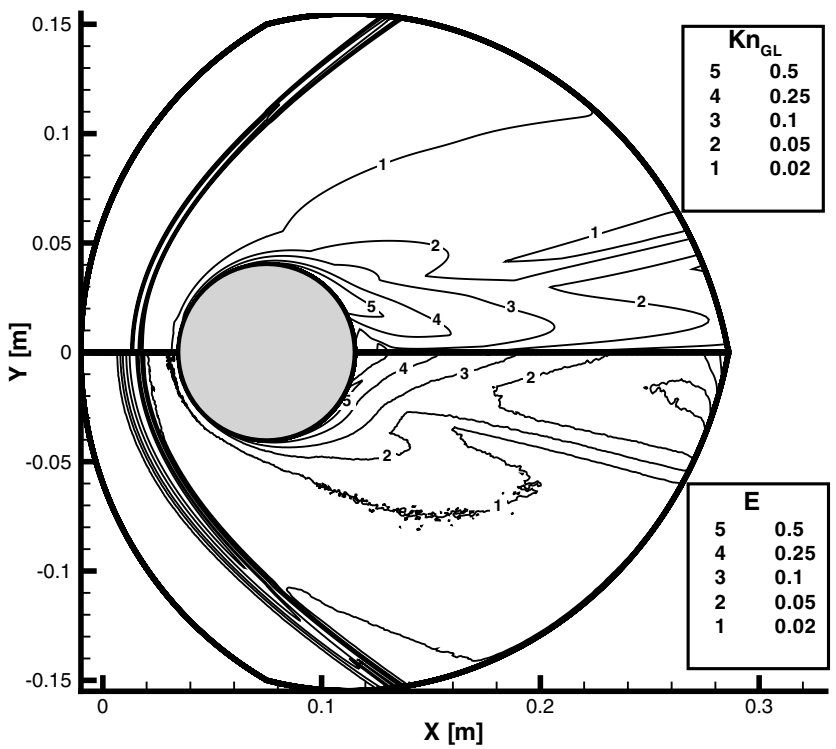

a) Continuum breakdown parameter is highest in the shock, forebody boundary layer, and near wake. Regions in which $K n_{\mathrm{GL}}$ is large are seen to correspond very well with regions of high error $E$. In the near-wake region, the error in the NS solution is seen to range from 10 to $40 \%$. The dominant error in this region is determined to be the velocity magnitude, which would likely improve with the use of slip boundary conditions in the NS solver. Quantitatively, Fig. 1a reveals that the breakdown parameter predicts the magnitude of this error quite well. More specifically, $K n_{\mathrm{GL}}$ is found to uniformly overpredict the error by $40 \%$. That is, the contours of $K n_{\mathrm{GL}}$ quite closely match the contours of $1.4 \times E$. Similar agreement is found for cases M3 and M12. These results lend further support to previous recommendations $[16,22]$ of setting breakdown cutoff at $K n_{\mathrm{GL}}=0.05$. Thus, any cell in which the value of $K n_{\mathrm{GL}}>0.05$ is set as a particle (DSMC) cell and the remaining cells are set as continuum (NS) cells. The initial and final interface locations for this case (M6) are shown in the top portion of Fig. 1b. The initial interface corresponds to contour level 2 in the top of Fig. 1 a after a simple smoothing algorithm was applied. The letters a through $\mathbf{d}$ denote regions of interest for later reference. The bottom portion of Fig. 1b shows the final interface locations for all three cases presented in this paper. Clearly, the higher the flow speed, the larger the nonequilibrium particle region. Note how the particle region ends before the domain exit for case $\mathbf{M 3}$ and how the shock and boundary-layer particle regions merge for the M12 case.

\section{MPC Coupling Method}

The modular implementation of the MPC method allows both DSMC and NS solvers to load and store the same mesh in their own separate data structures. This involves duplication of the mesh geometry but allows both solvers to operate on their own data structure without modification. Each cell of one mesh is simply linked to the corresponding cell in the other mesh and can easily access its information. Cells labeled as particle cells are simply not used in the NS mesh and, likewise, those labeled as continuum cells will contain no particles in the DSMC mesh. This implementation is depicted in Fig. 2, in which the cells with dotted lines are stored in memory but are not used. After application of the breakdown parameter $K n_{\mathrm{GL}}$, the particle region is immediately extended by a few extra cells into the continuum domain to create an overlap region. Next, one row of NS boundary cells and two rows of DSMC boundary cells are initialized, as seen in Fig. 2 . Now that all regions of NS and DSMC cells, including boundary cells, have been defined, the regions must be coupled by transferring information across the interface. This is handled by simply updating the conditions inside

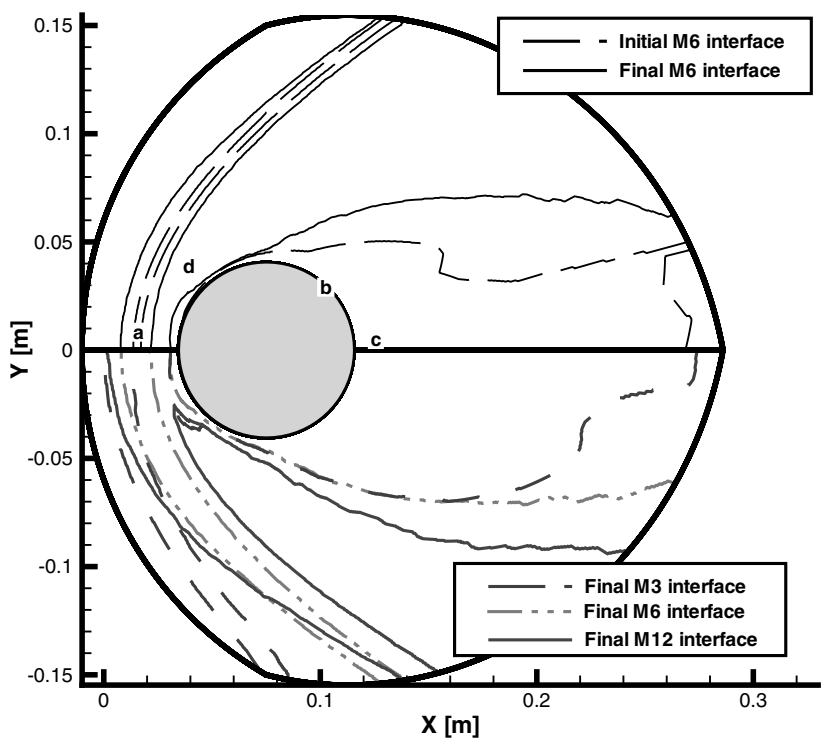

b) DSMC-NS interface locations

Fig. 1 Continuum breakdown and interface locations. 


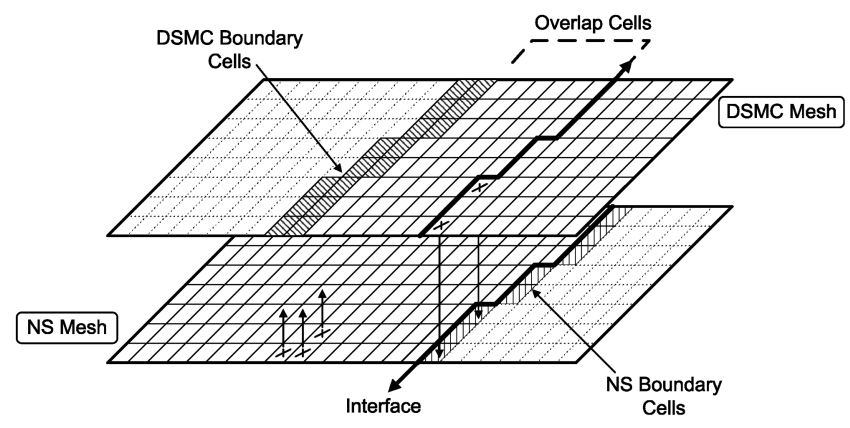

Fig. 2 State-based coupling.

boundary cells and employing the standard boundary procedures already used by both solvers.

To transfer information from NS to DSMC regions, DSMC boundary cells are continually filled with particles consistent with the flow properties in the corresponding NS cells. For each time step, all simulation particles in the DSMC boundary cells are first deleted and then regenerated based on current NS information. The number of new particles is determined directly from the NS cell density, and the particles are randomly distributed within the DSMC boundary-cell volume. The velocities of these newly generated particles are sampled from a Chapman-Enskog distribution [23] based on the local macroscopic state and gradients, known from the NS solver. The boundary cells then become an extension of the DSMC domain for one standard DSMC cycle. As particles in the DSMC domain interact and their distributions evolve in time, the MPC method also tracks the macroscopic variation in each DSMC cell. To provide these averaged properties with minimal statistical scatter, a mixture of spatial and temporal averaging is used. Specifically, this study uses the subrelaxation technique proposed by Sun and Boyd [24], which is able to provide low-scatter macroscopic quantities in each DSMC cell at each time step. When transferring information from DSMC to NS regions, these averaged DSMC properties can then be used to set the conditions in the corresponding NS boundary cells.

The MPC method begins with a full NS solution to the problem and uses the continuum breakdown parameter to determine initial DSMC and NS regions. Particles are initialized in DSMC regions such that they are consistent with the NS solution. As the DSMC module iterates in particle regions, the solution proceeds toward the correct nonequilibrium solution. This change is transferred to continuum regions by updating the NS boundary conditions, as described earlier. As the solution evolves, the MPC numerical cycle continues to loosely couple the two solvers while adaptively repositioning the interfaces using the continuum breakdown parameter. At some point, the DSMC and NS solutions will reach a steady state. Further application of the continuum breakdown parameter will not modify the interface position, and interfaces can thus be locked in place. After this steady state has been reached, the MPC method proceeds to both sample the DSMC regions and converge the NS regions. As continued sampling reduces the scatter in the DSMC regions, any scatter transferred to the NS regions is also reduced. The result is a scatter-free DSMC solution in nonequilibrium regions that transitions to a converged NS solution in continuum regions. A complete explanation of this coupling procedure and numerical cycle is contained in [17].

\section{Numerical Investigation of Nonequilibrium Regions}

\section{A. Velocity Distribution Functions}

To investigate the degree of nonequilibrium in hypersonic bluntbody flowfields, it is instructive to look at the local velocity distribution function (VDF). The MPC solver uses DSMC in nonequilibrium regions and the NS equations in near-equilibrium regions in which the local VDF should be a Chapman-Enskog distribution. Thus, an MPC simulation contains all of the information required to generate the physically correct VDF at every location in the flow. The local VDFs for four regions of interest (labeled a through $\mathbf{d}$ in Fig. 1b) are generated using full DSMC, full NS, and
MPC simulations for case M6 and are shown in Fig. 3 . Here, the probability density function (PDF) of a particle having a certain $x$ velocity $V_{x}$ or $y$ velocity $V_{y}$ is plotted. The first observation from all VDFs plotted in Fig. 3 is that the MPC particles do indeed have the same velocity distribution as particles within a full DSMC simulation. The most severe nonequilibrium region is in the shock center along the stagnation streamline (region a), shown in Fig. $3 \mathrm{a}$. To adjust for the different shock positions between DSMC and $\overline{\mathrm{NS}}$ solutions, the shock center is taken as the point at which $T_{\mathrm{TRA}}=$ $0.5\left(T_{\text {TRA post }}+T_{\text {TRA pre }}\right)$ for DSMC and MPC and at which $T=$ $0.5\left(T_{\text {post }}+T_{\text {pre }}\right)$ in the NS solution. The dotted lines shown in Fig. 3 are Chapman-Enskog distributions generated [23] using the local NS state and gradients. In Fig. $3 \mathrm{a}$, the VDF in the $x$ direction shows a peak of fast-moving particles, but also a significant number of postshock particles that have traveled upstream through the thin shock and maintained their low speed. The Chapman-Enskog distribution effectively assumes more collisions and thus the fastmoving peak is lowered and the slow-moving region of the VDF is raised. Certainly, the NS equations do not contain the information needed to describe the VDF in either the $x$ or $y$ direction inside the shock. Another region of the flow that is highly nonequilibrium is region $b$, located $135 \mathrm{deg}$ around the cylinder surface. The local VDFs are plotted in Fig. $3 \mathrm{~b}$, in which the Chapman-Enskog VDFs are centered around zero velocity due to the no-slip condition in the NS solver. In addition to the significant velocity slip evident in the DSMC and MPC VDFs, Fig. $3 b$ reveals that the VDF in the $y$ direction is quite far from having an equilibrium shape. The local VDF in a cell $2 \mathrm{~cm}$ directly behind the cylinder (region c) is plotted in Fig. 3c. Here, the DSMC and MPC VDFs appear quite similar to the Chapman-Enskog distribution from the NS solution, only they are slightly wider. It will be shown in the next section that this width does indeed correspond to a higher temperature in this region. Finally, for region $\mathbf{d}$, which lies downstream of the shock but away from the surface, Fig. $3 \mathrm{~d}$ shows that the DSMC distribution is indeed a Chapman-Enskog distribution. In addition, this is a continuum region of the mesh, in which the MPC method solves the NS equations. Figure $3 \mathrm{~d}$ demonstrates that the NS equations are sufficient to represent the local VDF in this region. It should be noted that because of the large velocity scales in these VDF plots, it is difficult to determine the degree to which small variations in a VDF translate into noticeable macroscopic effects. For this reason, the flowfield and surface properties are now investigated.

\section{B. Forebody and Near-Wake Flowfields}

The purpose of the MPC method is ultimately to reproduce the flowfield and surface property results of a full DSMC simulation in less time. The $T_{\text {TRA }}$ fields calculated by full DSMC, full NS, and MPC methods are compared in Fig. 4 for the M12 case. Compared with the NS shock profile, the DSMC shock wave is significantly thicker and begins further upstream. In addition, the translational temperature overshoot is clearly visible in the DSMC solution. The wake region also shows that the temperature predicted by DSMC is approximately $10-15 \%$ higher than that predicted by the NS solver. Qualitatively, very similar results are obtained for cases M3 and M6. Figure 4 also shows that the MPC method successfully reproduces a full DSMC solution with little error. In fact, the largest error at any location in the flow for any flow variable [see Eq. (4), replacing $Q_{\mathrm{NS}}$ with $Q_{\mathrm{MPC}}$ ] is determined to be less than $3.0,2.5$, and $1.5 \%$ for cases M12, M6, and M3, respectively. Moreover, the largest error is in the $T_{\text {TRA }}$ field, which is maximum in MPC-continuum regions (away from the surface), in which the NS equations do not model thermal nonequilibrium. This may be quite acceptable, because conditions in the boundary-layer and near-wake regions are of most importance. The MPC solver correctly models these regions as particle regions in which DSMC results are reproduced almost exactly.

A more detailed comparison is shown in Fig. 5a, in which both the translational and rotational temperature profiles are plotted for each method along the 60-deg line shown in Fig. 4. In this region, the flow begins to expand immediately after passing through the shock wave. 


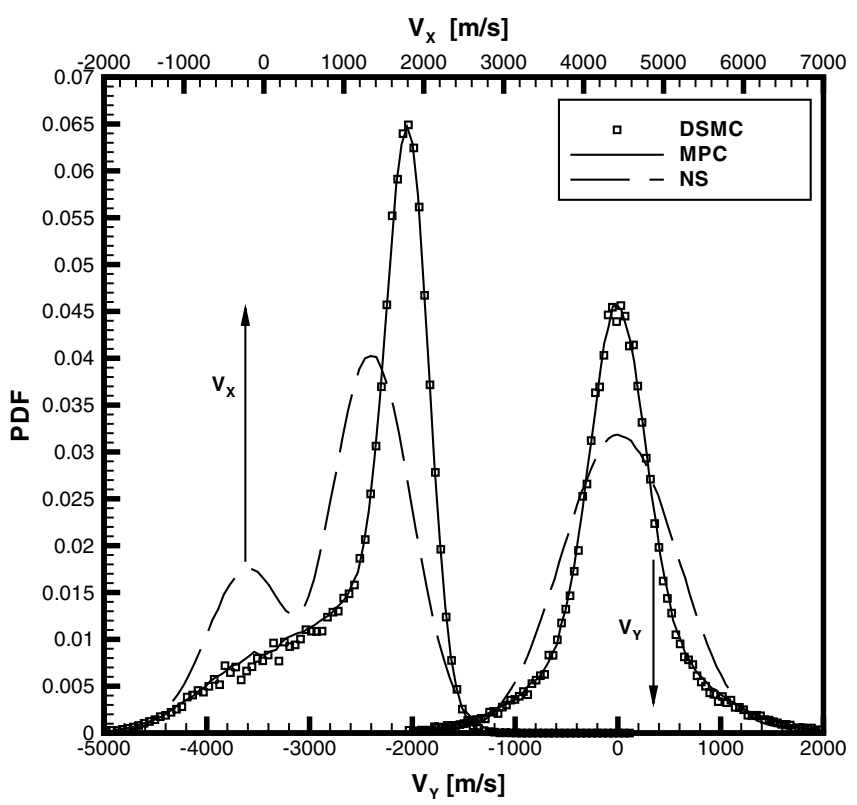

a) Local VDF in shock center

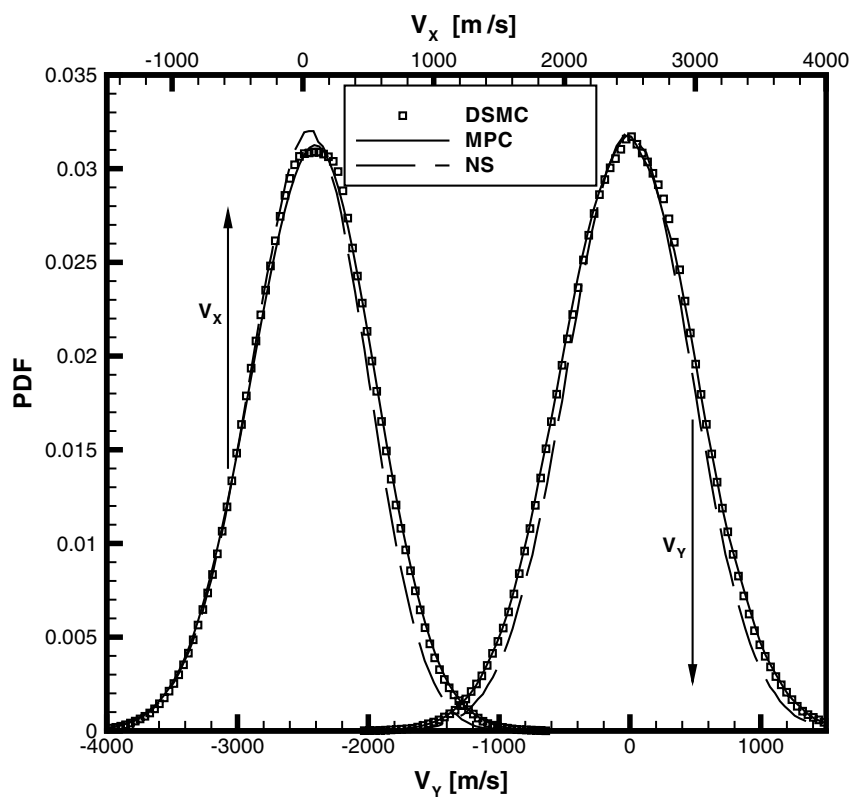

c) Local VDF in near-wake region

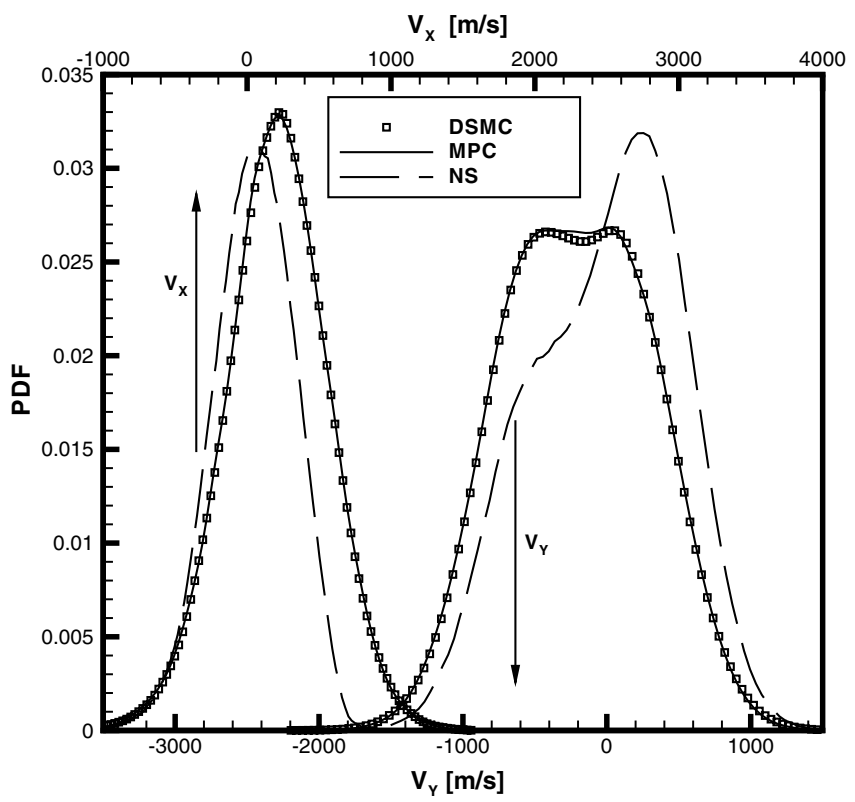

b) Local VDF at $135 \mathrm{deg}$ on surface

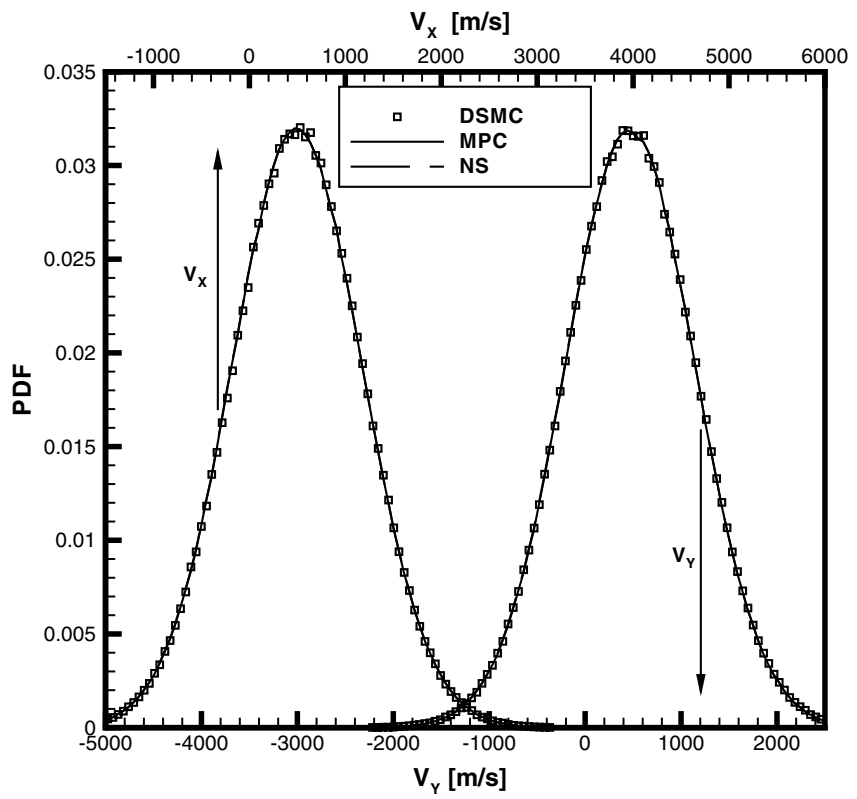

d) Local VDF in postshock continuum region

Fig. 3 Local velocity distribution functions for case M6.

Because rotational energy requires more collisions to equilibrate than the translational energy, it remains frozen at a higher rotational temperature in this expanding region of the flow. Focusing only on the DSMC results, this behavior can be seen in Fig. 5a. Moving focus to the MPC results, Fig. 5a shows that the MPC method predicts the correct profiles for both $T_{\mathrm{TRA}}$ and $T_{\mathrm{ROT}}$ in both the shock and boundary-layer particle regions. However, in the MPC-continuum region between these interfaces, the NS equations are solved that use a single temperature combining both translational and rotational modes. As expected, the MPC temperature profile in this region lies between the $T_{\text {TRA }}$ and $T_{\text {ROT }}$ profiles predicted by DSMC. Finally, focusing on the full NS solution in Fig. 5a, it is important to notice that at the interface just after the shock, the MPC temperature is not equal to the initial NS temperature. This means that the MPC cycle has succeeded in providing more accurate boundary conditions to the MPC-continuum region. These conditions were set (or transferred) using the MPC-particle region and result in shifting the MPCcontinuum profile to a lower, more accurate, translational temperature. However, in this case, the shift is not significant and begs the question: If the postshock state for both DSMC and NS solutions is virtually the same, is it even necessary to accurately predict the shock interior? To answer this question, two additional MPC simulations are tested. The first, denoted as M12a, only models the boundary layer and wake as particle regions. That is, the shock is simulated as a continuum region in which the NS equations are solved. This approach may seem similar to the zonally decoupled solutions discussed earlier [3-5] ; however, the MPC method adapts the interface locations and restricts particle regions only to that portion of the flowfield in which large nonequilibrium effects are felt. For the M12 case, these regions include the forebody boundary layer and only a portion of the wake. The second simulation, denoted as M12b, models only the shock with a particle region and solves the NS equations everywhere else. Both cases use the exact same flow conditions, mesh, and numerical parameters as the M12 case and only differ in the final MPC interface locations. The resulting translational temperature profiles along the same 60-deg cut are shown in Fig. 5b. Although case M12a does not predict the correct shock profile, because it models the nonequilibrium boundary layer 


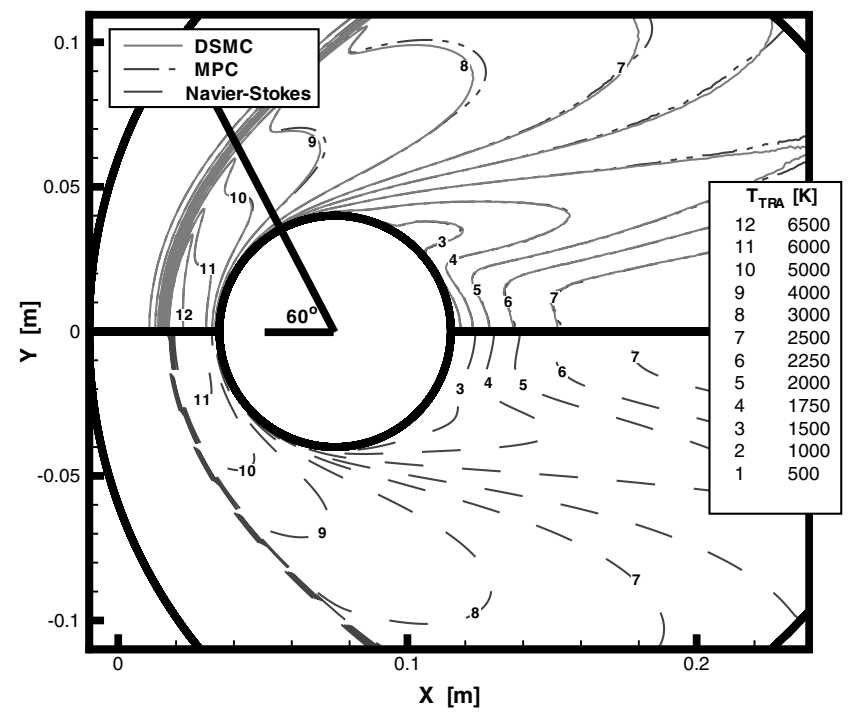

Fig. 4 Mach 12 translational temperature field.

as a particle region, it agrees well with DSMC results near the surface. Conversely, although case M12b predicts the correct shock profile, because the postshock state is only slightly different, the rest of the solution agrees very well with a full NS simulation. Near the cylinder surface, a continuum solution (such as case M12b) predicts steeper gradients that may influence the predicted heat transfer and momentum transfer to the spacecraft. Thus, based on the forebody flowfield, modeling the shock wave as a particle region may not be necessary. This will be verified in the next section by examining the predicted surface properties over the entire cylinder.

Nonequilibrium effects in the near-wake region are portrayed in Fig. 6. The $x$-velocity profiles along the symmetry axis, directly in the wake of the cylinder, are plotted in Fig. 6a for the M3, M6, and M12 cases. Here, the location at which the $x$ velocity becomes positive indicates the location of wake closure. For each freestream Mach number, DSMC simulations predict wake closure to occur closer to the cylinder than that predicted by a full NS simulation. In addition, higher Mach number flow, associated with larger nonequilibrium effects, is observed to result in a smaller wakeclosure length. Both of these results agree qualitatively with those presented in [3], which compared DSMC and NS results for a slightly more complicated geometry at higher flow speeds. The temperature

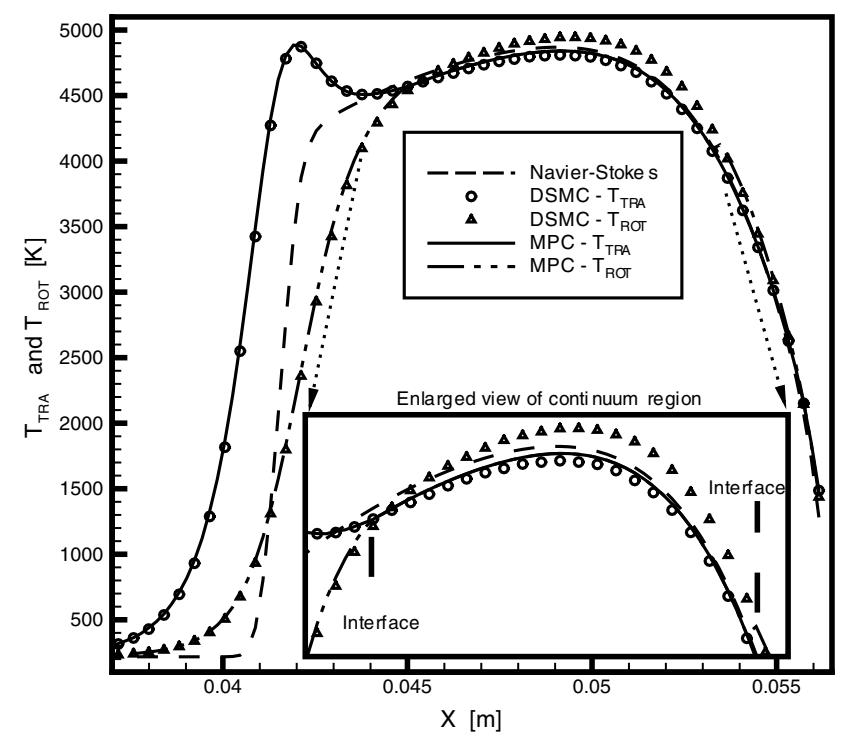

a) Thermal nonequilibrium effects profiles in the near-wake region are plotted in Fig. $6 \mathrm{~b}$ for all three cases. The wake flow is seen to be slightly out of thermal equilibrium. In addition, as seen previously in Fig. $\underline{4}$, the translational temperature predicted by DSMC is higher than that predicted by a NS solution. Although not shown, the density in this region is correspondingly lower for DSMC, whereas the pressure predicted by both NS and DSMC remain similar. Finally, for each of the near-wake results plotted in Fig. 6, the MPC method is seen to reproduce the DSMC profiles with little error. Although not shown, case M12a is verified to reproduce DSMC wake results, and case M12b reproduces NS wake results.

\section{Surface Properties}

This paper presents, for the first time in the literature, a detailed analysis of the surface properties predicted by a hybrid DSMC-NS solver. Figure 7 plots the coefficients of heat transfer $C_{q}$ and shear stress $C_{\tau_{\|}}$around the cylinder surface for cases M3, M6, M12, M12a, and M12b. The coefficients are defined as

$$
C_{q}=\frac{q}{\frac{1}{2} \rho_{\infty} u_{\infty}^{3}} \quad \text { and } \quad C_{\tau_{\|}}=\frac{\tau_{\|}}{\frac{1}{2} \rho_{\infty} u_{\infty}^{2}}
$$

where $q$ is the heat transferred to the cylinder per unit time per unit area and $\tau_{\|}$is the tangential momentum per unit time per unit area transferred to the cylinder at each point on the surface. In a NS simulation, both $q$ and $\tau$ are determined using gradients of macroscopic flow properties (temperature and velocity). In a DSMC simulation, $q$ and $\tau$ are determined by averaging changes in the kinetic energy and momentum of individual particles as they collide with the surface. In all MPC simulations, as depicted earlier in Fig. 1b, a particle region envelopes the entire cylinder surface. Thus, heat and momentum transfer in the MPC simulations is determined in the same way as in a full DSMC simulation. It is evident that for each flow Mach number in Figs. $7 \mathrm{a}-7 \mathrm{c}$, the heat and momentum transfer predicted by DSMC is less than that predicted by the NS solver around the entire cylinder. This is due in part to velocity slip and temperature jump at the surface, which is not modeled in the NS simulations but is a natural result of the DSMC simulations. However, it is also due to the fact that in assuming more collisionality, the NS equations predict more rapid flow changes (steeper gradients), which correspond to higher rates of heat and momentum flux. The discrepancy in the peak heating rate is seen to be 4.4, 10.0, and 7.5\% for cases M3, M6, and M12, respectively. The largest difference between DSMC and NS is in the wake of the cylinder, in which the shear stress is significantly less, according to

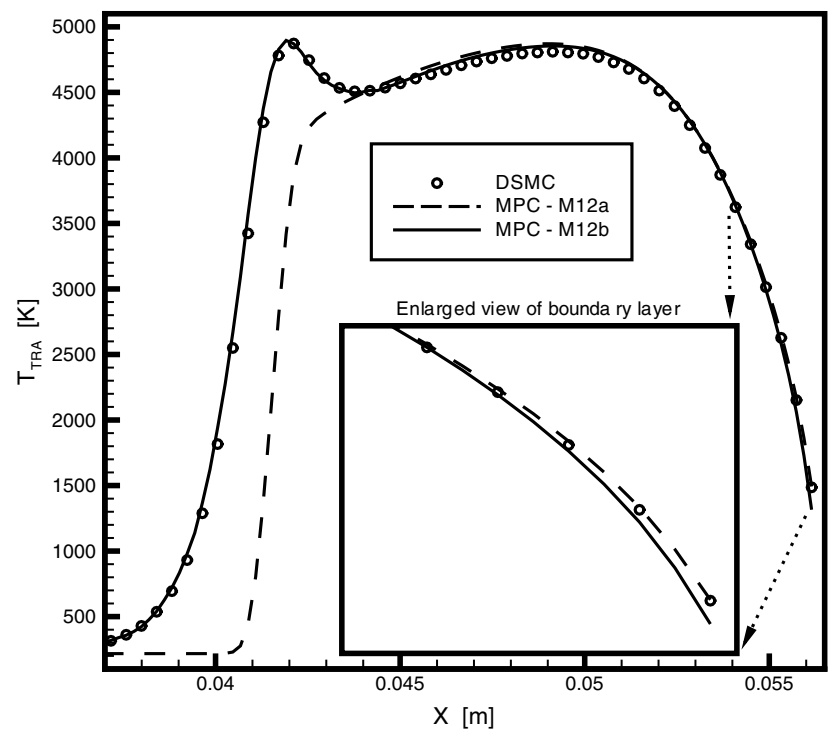

b) Effect of accurately solving the shock interior

Fig. 5 Forebody temperature profiles along a 60-deg cut. 


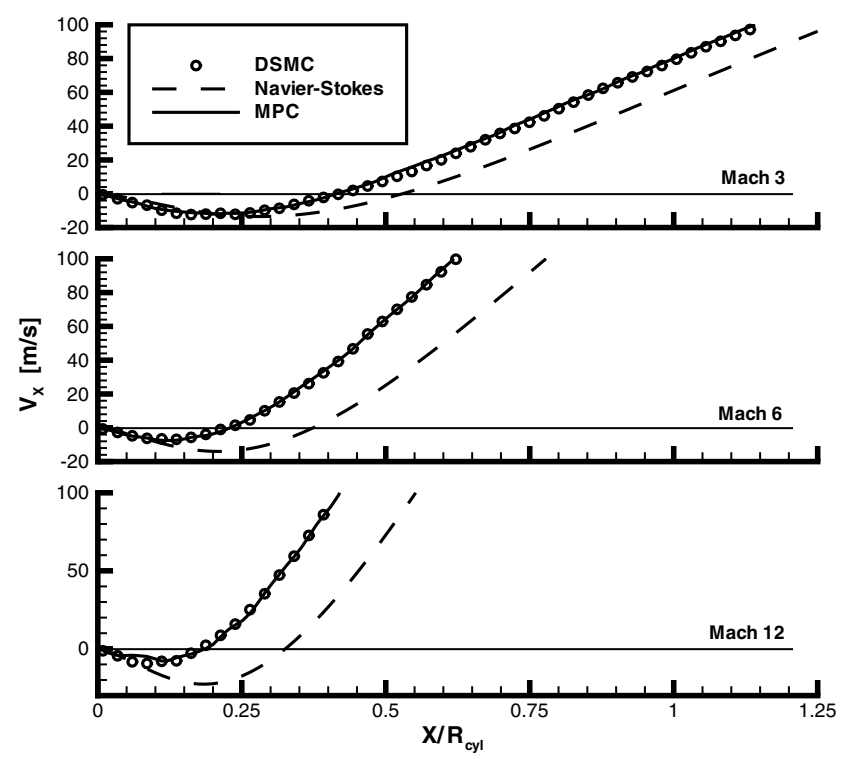

a) Wake closure
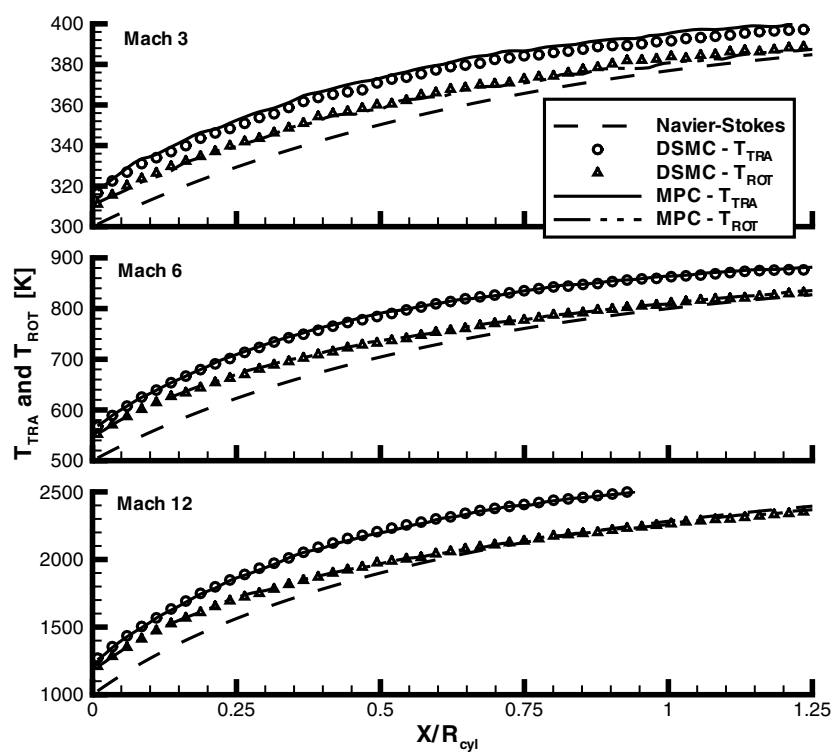

b) Near-wake temperature profile

Fig. 6 Nonequilibrium effects in the near-wake region.

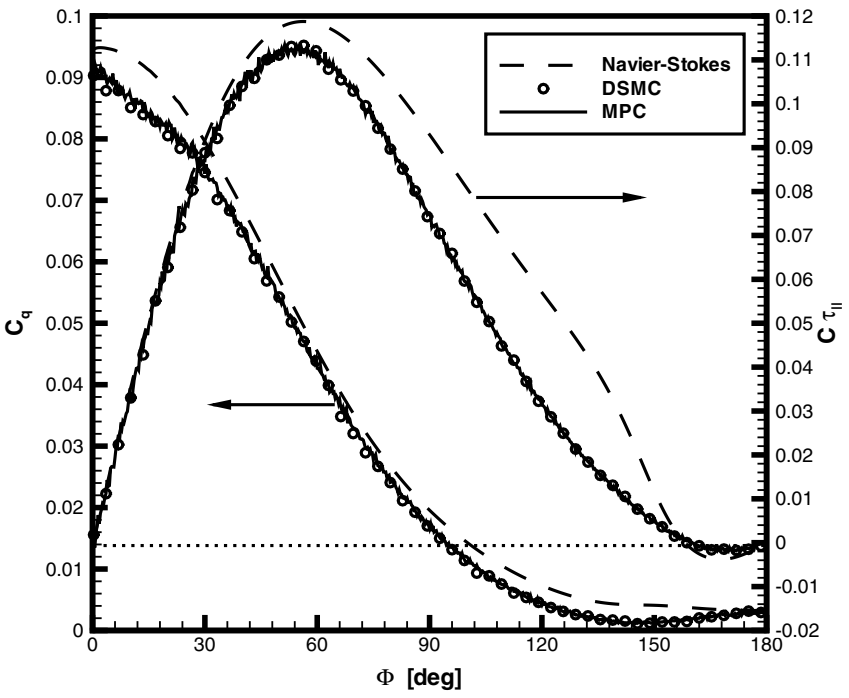

a) Mach 3 surface properties

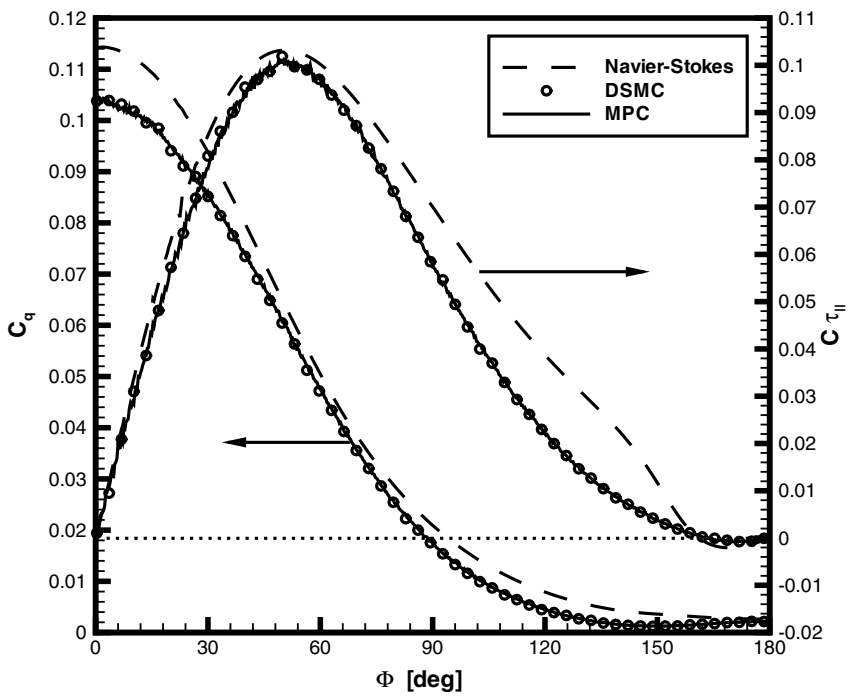

b) Mach 6 surface properties

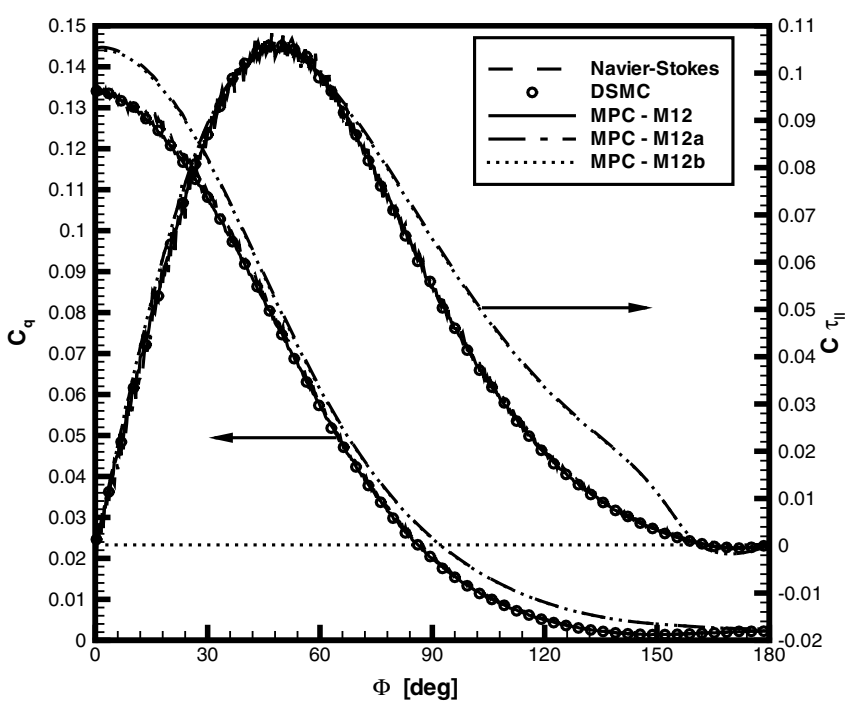

c) Mach 12 surface properties

Fig. 7 Heat transfer and shear stress surface properties. 
DSMC. These results are qualitatively and quantitatively similar to those presented in [25] for simulations of Mach 10 flow of argon around a cylinder. However, as shown in that study, the coefficient of pressure $C_{p}$ predicted by both NS and DSMC is virtually the same. Because the majority of drag on the cylinder is due to pressure drag, despite differences in shear stress, the total drag on the cylinder remains the same for both NS and DSMC. Again, Figs. 7a-7c demonstrate that the MPC method successfully reproduces $\overline{\mathrm{DS}} \overline{\mathrm{MC}}$ results. What is also evident in Fig. 7c is that when the shock is not modeled using a particle region (case M12a), the surface properties are still accurately predicted. In the forebody, up to an angle of approximately $30 \mathrm{deg}$, the heat transfer is seen to be slightly higher, but elsewhere the results are identical. Also as expected, the M12b case, which captures the shock accurately with a particle region but uses the NS equations everywhere else, predicts surface properties consistent with a full NS solver.

The computational efficiency gained by the MPC algorithm over pure DSMC and the precise manner in which MPC and DSMC simulation times are compared is detailed in [17]. As expected, the speedup gained by the MPC method is found to be directly proportional to the relative size of the continuum regions or, more specifically, proportional to the number of particles saved by restricting the use of DSMC to nonequilibrium regions only. For cases M3, M6, and M12, the MPC method uses 3.0, 2.4, and 1.65 times fewer particles and reaches the solution 2.2, 1.6, and 1.4 times faster, respectively, than full DSMC simulations. Analysis of the additional M12a case reveals that by not modeling the shock wave as a particle region, a large number of particles are eliminated because the shock is a dense region of the flow. The speedup achieved for the Mach 12 flow conditions is determined to increase from 1.4 to 2.8 times and, as shown earlier, aside from the internal shock structure, the MPC results for case M12a accurately reproduce full DSMC flowfield and surface properties.

\section{Conclusions}

In this paper, simple hypersonic blunt-body flowfields exhibiting a mixture of continuum and nonequilibrium flow were studied using DSMC, NS, and MPC numerical simulations. Analysis of local velocity distributions reveals that large regions of the flowfield, such as between the shock and boundary layer, contain near-equilibrium particle distributions that can be modeled accurately by the NS equations. At the same time, the velocity distributions inside the shock wave and in the near-wake region are found to deviate significantly from near-equilibrium (Chapman-Enskog) distributions. As a result, NS solutions become inaccurate in these regions. For 2-D flow of $\mathrm{N}_{2}$ over a cylinder at various Mach numbers for which the global Knudsen number is 0.01 , NS simulations significantly overpredict the local shear stress. However, this has a negligible effect on the total drag, because the predicted surfacepressure distributions from DSMC and NS are similar. In addition, the NS simulations are found to overpredict the peak heating rate by $5-10 \%$, predict slower wake closure, and predict $10-15 \%$ lower temperatures in the immediate wake region. The MPC code is able to accurately reproduce DSMC flowfield results in which the maximum error in any variable at any location is $3 \%$. This maximum error is in temperature and is found away from the cylinder surface in MPCcontinuum regions, in which the NS module does not account for thermal nonequilibrium. This error is acceptable, because the boundary-layer and near-wake regions are of more importance for the prediction of surface properties. For the first time, a hybrid particle-continuum numerical method is shown to be capable of reproducing the local velocity distribution functions and surface properties predicted by full DSMC simulations. The MPC simulation results demonstrate that solution of the NS equations is physically accurate in regions of the flowfield in which the gradient-length Knudsen number (with an added condition of thermal equilibrium) is less than 0.05. In addition, the gradient-length Knudsen number is shown to provide a reasonable prediction for the magnitude of discrepancy between NS and DSMC solutions. The MPC method is shown to reproduce DSMC results for hypersonic flow about a 2-D cylinder $(K n=0.01)$ with speedup factors of $1.4,1.6$, and 2.2 for freestream Mach numbers of 12, 6, and 3, respectively. The computational time saved by the MPC method is directly proportional to the fraction of the flowfield, which is in near equilibrium. It is found that particle simulation of the shock interior is not necessary for accurate prediction of surface properties, but particle simulation of the boundary-layer and near-wake regions is necessary. When an MPC simulation of Mach 12 flow is repeated without particle simulation of the shock wave, the speedup factor rises from 1.4 to 2.8 with no noticeable loss of accuracy.

\section{Acknowledgments}

This work is sponsored by the Space Vehicle Transportation Institute under NASA grant NCC3-989 with joint sponsorship from the U.S. Department of Defense and under the U.S. Air Force Office of Scientific Research grant FA9550-05-1-0115. This work is also supported by the Francois-Xavier Bagnoud Foundation.

\section{References}

[1] Bird, G. A., Molecular Gas Dynamics and the Direct Simulation of Gas Flows, Oxford Univ. Press, New York, 1994.

[2] Moss, J. N., and Price, J. M., "Survey of Blunt Body Flows Including Wakes at Hypersonic Low-Density Conditions," Journal of Thermophysics and Heat Transfer, Vol. 11, No. 3, 1997, pp. 321-329.

[3] Wilmoth, R. G., Mitcheltree, R. A., Moss, J. N., and Dogra, V. K., "Zonally Decoupled Direct Simulation Monte Carlo Solutions of Hypersonic Blunt-Body Wake Flows," Journal of Spacecraft and Rockets, Vol. 31, No. 6, 1994, pp. 971-979.

[4] Glass, C. E., and Gnoffo, P. A., "A 3-D Coupled CFD-DSMC Solution Method with Application to the Mars Sample Return Orbiter," NASA TM-2000-210322, July 2000.

[5] Glass, C. E., and Horvath, T. J., "Comparison of a 3-D CFD-DSMC Solution Methodology with a Wind Tunnel Experiment," NASA TM2002-211777, Aug. 2002.

[6] Wijesinghe, H. S., and Hadjiconstantinou, N. G., "Discussion of Hybrid Atomistic-continuum Methods for Multiscale Hydrodynamics," International Journal of Multiscale Computational Engineering, Vol. 2, No. 2, 2004.

[7] Hash, D. B., and Hassan, H. A., "Assessment of Schemes for Coupling Monte Carlo and Navier-Stokes Solution Methods," Journal of Thermophysics and Heat Transfer, Vol. 10, No. 2, 1996, pp. 242249.

[8] Hash, D. B., and Hassan, H. A., "A Decoupled DSMC/Navier-Stokes Analysis of a Transitional Flow Experiment," 34th AIAA Aerospace Sciences Meeting and Exhibit, Reno, NV, AIAA Paper 96-0353, Jan. 1996.

[9] Wijesinghe, H. S., Hornung, R. D., Garcia, A. L., and Hadjiconstantinou, N. G., "Three-Dimensional Hybrid ContinuumAtomistic Simulations For Multiscale Hydrodynamics," Journal of Fluids Engineering, Vol. 126, No. 5, 2004, pp. 768-777.

[10] Hash, D. B., and Hassan, H. A., "Two-Dimensional Coupling Issues of Hybrid DSMC/Navier-Stokes Solvers," 32nd AIAA Thermophysics Conference, Atlanta, GA, AIAA Paper 97-2507, June 1997.

[11] Roveda, R., Goldstein, D. B., and Varghese, P. L., "Hybrid Euler/ Particle Approach for Continuum/Rarefied Flows," Journal of Spacecraft and Rockets, Vol. 35, No. 3, 1998, pp. 258-265.

[12] Roveda, R., Goldstein, D. B., and Varghese, P. L., "Hybrid Euler/Direct Simulation Monte Carlo Calculation of Unsteady Slit Flow," Journal of Spacecraft and Rockets, Vol. 37, No. 6, 2000, pp. 753-760.

[13] Wang, W.-L., and Boyd, I. D., "Hybrid DSMC-CFD Simulations of Hypersonic Flow over Sharp and Blunted Bodies," 36th AIAA Thermophysics Conference, Orlando, FL, AIAA Paper 03-3644, 2003.

[14] Sun, Q., and Boyd, I. D., "A Direct Simulation Method for Subsonic, Microscale Gas Flows," Journal of Computational Physics, Vol. 179, No. 2, 2002, pp. 400-425.

[15] Wang,W.-L., and Boyd, I. D., "A New Energy Flux Model in the DSMC-IP Method for Nonequilibrium Flows," AIAA Thermophysics Conference, Orlando, FL, AIAA Paper 03-3774, 2003.

[16] Schwartzentruber, T. E., and Boyd, I. D., "A Hybrid ParticleContinuum Method Applied to Shock Waves," Journal of Computational Physics, Vol. 215, No. 2, 2006, pp. 402-416.

[17] Schwartzentruber, T. E., Scalabrin, L. C., and Boyd, I. D., "A Modular Particle-Continuum Numerical Method for Hypersonic Nonequilibrium Flows," Journal of Computational Physics (to be published). 
[18] Dietrich, S., and Boyd, I. D., "Scalar and Parallel Optimized Implementation of the Direct Simulation Monte Carlo Method," Journal of Computational Physics, Vol. 126, No. 2, 1996, pp. 328-342.

[19] Boyd, I. D., "Analysis of Rotational Nonequilibrium in Standing Shock Waves of Nitrogen," AIAA Journal, Vol. 28, No. 11, 1990, pp. 1997 1999.

[20] Scalabrin, L. C., and Boyd, I. D., "Development of an Unstructured Navier-Stokes Solver for Hypersonic Nonequilibrium Aerothermodynamics," 38th AIAA Thermophysics Conference, Toronto, Ontario, Canada, AIAA Paper 05-5203, 2005.

[21] MacCormack, R. W., and Candler, G. V., "The Solution of the Navier-Stokes Equations Using Gauss-Seidel Line Relaxation," Computers and Fluids, Vol. 17, No. 1, 1989, pp. 135-150.
[22] Boyd, I. D., Chen, G., and Candler, G. V., "Predicting Failure of the Continuum Fluid Equations in Transitional Hypersonic Flows," Physics of Fluids, Vol. 7, No. 1, 1995, pp. 210-219.

[23] Garcia, A. L., and Alder, B. J., "Generation of the Chapman-Enskog Distribution," Journal of Computational Physics, Vol. 140, No. 1 , 1998, pp. 66-70.

[24] Sun, Q., and Boyd, I. D., "Evaluation of Macroscopic Properties in the Direct Simulation Monte Carlo Method," Journal of Thermophysics and Heat Transfer, Vol. 19, No. 3, 2005, pp. 329-335.

[25] Lofthouse, A. J., Boyd, I. D., and Wright, M. J., "Effects of Continuum Breakdown on Hypersonic Aerothermodynamics," 44th AIAA Aerospace Sciences Meeting and Exhibit, Reno, NV, AIAA Paper 06993, Jan. 2006. 